\title{
Mapeo y amenazas de los servicios ecosistémicos culturales de la pesca artesanal para el desarrollo regional en una subcuenca antropizada del occidente de México
}

Eréndira Canales-Gómez. Universidad de Guadalajara, Puerto Vallarta, México. Romina Díaz-Gómez. Universidad Nacional de La Plata, La Plata, Argentina. José Cervantes-Ríos. Universidad de Guadalajara, Puerto Vallarta, México. Jorge Téllez-López. Universidad de Guadalajara, Puerto Vallarta, México.

RESUMEN | Los pescadores artesanales reciben de la naturaleza servicios ecosistémicos culturales (sc) que son esenciales para su bienestar y que pueden afectarse por intervenciones de desarrollo que ignoran su importancia y ubicación. En este estudio cuantificamos la relevancia de estos SC, analizando las relaciones espaciales de su flujo e identificando las amenazas que los comprometen, a través de encuestas, entrevistas semiestructuradas, mapeo participativo y valoración social a pescadores de la subcuenca del río Mascota, estado de Jalisco, México. Los análisis revelaron que los SC integran la identidad colectiva e individual de los pescadores y ocurren en los ríos y cuerpos de agua próximos a las localidades principales. Las amenazas fueron disminución de la abundancia de peces y captura debido a malas prácticas, alteraciones de los ríos y pérdida del bosque de ribera. Esta investigación visibiliza el valor de los SC y propone acciones para su inclusión en los planes regionales de desarrollo.

PALABRAS CLAVE | desarrollo regional y local, medioambiente, espacio público.

ABSTRACT | Artisanal fishers receive cultural ecosystem services (CES) from nature, essential to their well-being, and can be affected by development policies neglecting their importance and spatial distribution. In this research, we quantify the social valuation of CES and analyze the spatial relationships of their flow and threats. Surveys and semi-structured interviews, participatory mapping, and social valuation were applied to fishers of the Mascota River subwatershed, Jalisco state, Mexico. We found that CES are incorporated into fisher's collective and individual identities and occur in rivers and water bodies near principal localities. The identified threats were the decreasing of fish abundance and artisanal catches caused by poor fisheries practices, along with river alterations and the loss of riparian forest. This research highlights the importance of CES and proposes actions for their inclusion in the regional development plans.

KEYWORDs | regional and local development, environment, public space. 


\section{Introducción}

La pesca artesanal es un medio por el cual personas de países en desarrollo y zonas rurales reciben servicios ecosistémicos ${ }^{1}$ de los ambientes de agua dulce, que constituyen una fuente importante de empleo, ingresos, seguridad alimentaria y beneficios inmateriales (Food and Agricultural Organization [FAO], 2018; High Level Panel of Experts [HLPE], 2014; Lynch et al., 2019). El estudio de las pesquerías artesanales y sus servicios ecosistémicos se ha centrado principalmente en dos temas: suministro de alimentos (Olaya-Rodríguez et al., 2017) y pesca recreativa como servicio cultural (Arlinghaus et al., 2017; Villamagna et al., 2014). Sin embargo, existen otros beneficios inmateriales y valores relacionales ${ }^{2}$ generados mediante la pesca que han sido escasamente abordados (Lynch et al., 2019; Small et al., 2017). Entre ellos, los asociados al sentido de pertenencia y patrimonio (Chan et al., 2012; Lynch et al., 2016) y los beneficios tangibles (uso artístico y ceremonial de los peces) e intangibles, como los derivados del placer de realizar la acción de pescar (beneficios estéticos, de actividad, de salud y espirituales) (Chan et al., 2012). El estudio de estos beneficios o servicios culturales (en adelante sc) es prioritario por diversas razones. Los SC son esenciales para el bienestar humano (Cooke et al., 2016; Lynch et al., 2016; MA, 2005). Figuran entre los valores más importantes que las personas asocian con la naturaleza (FAO, 2016), llegando a importar más que utilidades materiales afiliadas a la pesca, como dinero; y cambios físicos deseables, como el sustento o la vivienda (Chan et al., 2012). Son dependendientes de las percepciones sociales sobre los ecosistemas y sus componentes (Balvanera et al., 2009) y son difíciles de identificar explícitamente en el contexto espacial. Además, por su naturaleza intangible, presentan desafíos para su valoración y para la identificación de sus distintos beneficiarios a diferentes escalas (Small et al., 2017). Esto ha derivado en que sean subrepresentados en las evaluaciones de sc y en la planeación de políticas territoriales y toma de decisiones (Hernández-Morcillo et al., 2013).

Aunado a lo anterior, la continuidad de los sc derivados de la pesca está amenazada, como consecuencia de los efectos de las actividades humanas que han deteriorado y suprimido pesquerías continentales en países en desarrollo, como México (GasparDillanes \& Hernández-Montaño, 2013; Lyons et al., 1998), Brasil (Hallwass et al., 2013) y a nivel global (Cooke et al., 2016; FaO, 2018; Lynch et al., 2016, 2019). Por ejemplo, en México la construcción de presas ha propiciado el desarrollo de la pesca en zonas donde no era tradicionalmente importante, derivando en su incorporación como un componente relevante a nivel alimentario, socioeconómico y político de las comunidades (Gaspar-Dillanes \& Hernández-Montaño, 2013). En contraste, se

1 Los servicios ecosistémicos son aquellos beneficios que la población obtiene de los ecosistemas (Millennium Ecosystem Assessment [MA], 2005). Presentan las siguientes características: son beneficios (tangibles o intangibles) proporcionados espacial y heterogéneamente (flujos) como producto de las funciones o procesos de los ecosistemas; son aprovechados por determinados beneficiarios (de manera directa o indirecta) y, por lo tanto, son susceptibles de valorarse (monetaria o no monetariamente).

2 Los valores relacionales son aquellos que no emanan directamente de la naturaleza, pero que son derivados de nuestras relaciones con ella y nuestras responsabilidades hacia ella (Pascual et al., 2017). 
ha reportado disminución en los peces y sus pesquerías por la degradación ambiental asociada al crecimiento poblacional y expansión urbana (Lyons et al., 1998). Estos factores pueden generar cambios en los medios de vida de estas personas e impactos socioeconómicos para los pescadores artesanales de zonas rurales y periurbanas, principalmente (HLPE, 2014; Lynch et al., 2016; Mercado-Silva et al., 2011), que pueden resultar en ganancia o pérdida de sc. Estos factores priorizan la necesidad de evaluar los sc, incorporando a los beneficiarios dependientes de ellos y afectados por las amenazas y factores que puedan generar cambios en su suministro, especialmente a consecuencia de intervenciones de desarrollo (Ash et al., 2010; Beard et al., 2011).

El estrecho vínculo de las pesquerías continentales con casi la totalidad de los Objetivos del Desarrollo Sostenible (Cooke et al., 2016; faO, 2018), demanda a la vez una aproximación holística, desde el enfoque de los socioecosistemas (Arlinghaus et al., 2017; Beard et al., 2011), donde se valoren los beneficios inmateriales (Chan et al., 2012; Small et al., 2017). No obstante, esto puede ser un desafío en países latinoamericanos donde los datos son escasos (FAO, 2018; Hallwass et al., 2013; Olaya-Rodríguez et al., 2017), como México (Lyons et al., 1998), y donde se estima que no se reporta más del 60\% de las capturas (FAO, 2018). En estas situaciones, se ha sugerido emplear el conocimiento ecológico tradicional ${ }^{3}$ como un componente clave para la evaluación de sc (Beard et al., 2011; Díaz et al., 2015), debido a que dicho saber es la base de la subsistencia y mantenimiento de las pesquerías artesanales y sus servicios ecosistémicos (Berkes, 1999; Hallwass et al., 2013; Lynch et al., 2016).

Basados en lo expuesto, en este trabajo se analiza la pesquería artesanal de una región antropizada de México, con el objetivo de determinar su importancia en la provisión de SC para los pescadores artesanales; mapear la distribución espacial de su flujo e identificar sus amenazas, proveyendo información útil para su manejo y para la planificación, ordenamiento y desarrollo regional.

\section{Metodología}

\section{Área de estudio}

La subcuenca hidrológica del río Mascota $\left(2132 \mathrm{~km}^{2}\right)$ se localiza al occidente del estado de Jalisco, México; pertenece a la cuenca del río Ameca (Figura 1). Su población total (55.662 habitantes) se concentra principalmente en tres localidades: Ixtapa, Mascota y Talpa de Allende (84\%); el restante, en 206 ( $<2.500$ habitantes) (Instituto Nacional de Estadística y Geografía [INEGI], 2010). Las actividades económicas predominantes son agricultura, ${ }^{4}$ ganadería extensiva y explotación forestal (Instituto de Información Estadística y Geográfica [IIEG] Jalisco, 2017). Su red

3 Entendido como los conocimientos, prácticas y creencias que evolucionan a través de procesos adaptativos adquiridos del contacto humano directo con un ambiente específico y que son transmitidas mediante prácticas tradicionales (Berkes, 1999; Dudgeon \& Berkes, 2003).

4 Las prácticas agrícolas tradicionales de la región son los coamiles. Estos consisten en el cultivo de maíz principalmente en las laderas de los cerros, las cuales son desprovistas de vegetación. La práctica consiste en tres etapas: i) roza, tumba y quema de la vegetación, ii) siembra de maíz con coa, por periodo de dos a tres ańos, y iii) descanso de la parcela. 
hidrológica y cuerpos de agua favorecen la pesca artesanal a lo largo del río Mascota (corriente principal), afluentes y cuerpos de agua naturales (lagunas de: Juanacatlán, Yerbabuena y Potrerillos) y en el embalse artificial Presa Corrinchis, construido durante 1974-1977 con fines de riego agrícola. El proceso de antropización de la región está relacionado al cambio de uso de suelo (Instituto Nacional de Ecología y Cambio Climático [INECC]-Fondo Noroeste [FONNOR], 2018) y acelerada expansión urbana, principalmente en la parte baja (Baños Francia, 2018).

\section{FIGURA I | Subcuenca hidrológica del río Mascota. Se muestran los sitios muestreados en este estudio, localidades principales y uso de suelo urbano y agrícola}

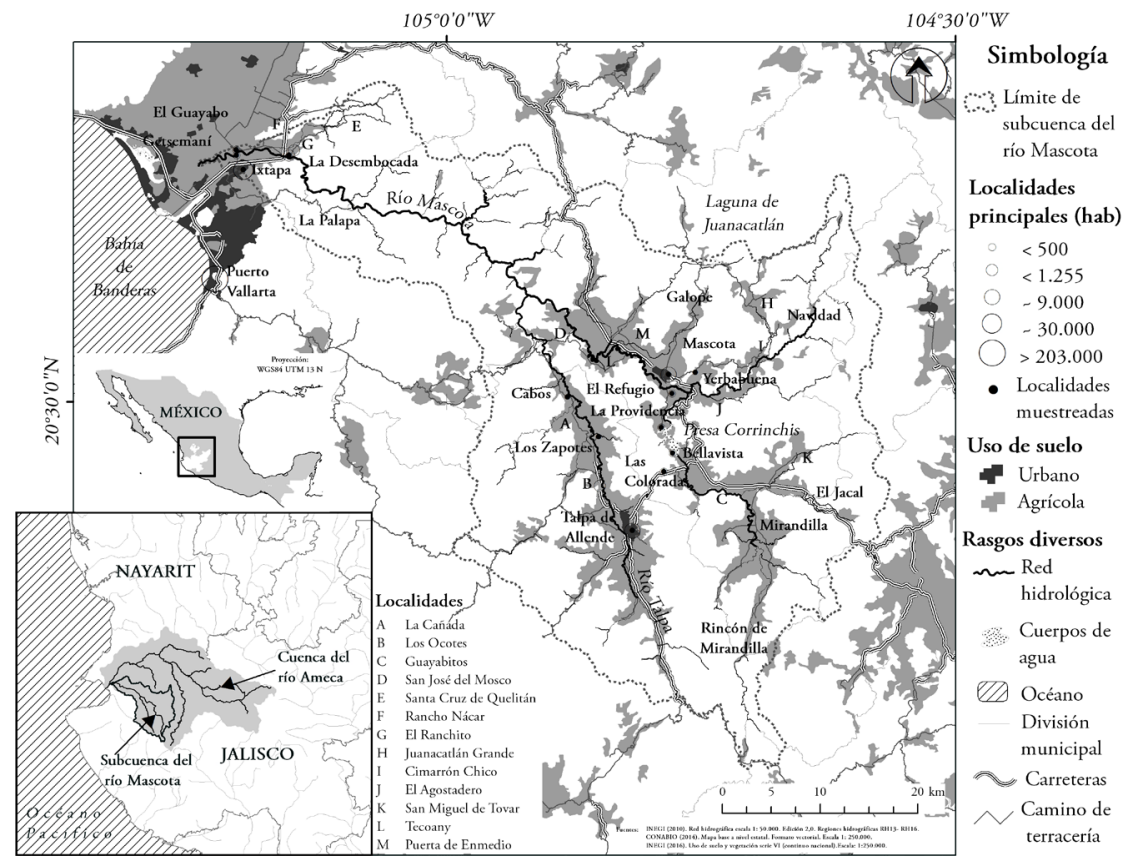

FUENTE: ELABORACIÓN PROPIA

\section{Diseño de la investigación}

Con base en los objetivos señalados, se planteó un diseño mixto de investigación. La importancia de los SC se evaluó adaptando el método de valoración social propuesto por Felipe-Lucia et al. (2015), consistente en entrevistar a los beneficiarios para conocer los sc que reciben, su valoración mediante escala Likert y los factores que los afectan. Esto se complementó con una encuesta para describir el perfil del pescador artesanal y caracterizar la pesquería de la región. Para el análisis se realizó un codificado de datos a priori, que fue complementado a posteriori mediante la identificación de temas de las entrevistas (Auerbach \& Silverstein, 2003). La distribución espacial del flujo de sc se determinó con análisis de densidad Kernel basado en 
datos obtenidos por mapeo participativo (Raymond et al., 2009). Una descripción detallada de las técnicas empleadas se presenta en los siguientes apartados.

El tipo de muestreo empleado fue no probabilístico por conveniencia, en combinación con el método de bola de nieve e informantes clave (Hernández-Sampieri et al., 2014; Mendieta-Izquierdo, 2015). El trabajo de campo se realizó en lugares de pesca (16) y domicilios particulares (30) de 15 localidades en la subcuenca, durante múltiples visitas (10), en los meses de julio a septiembre del ańo 2018. Se entrevistó y encuestó a 46 personas ( 45 hombres y una mujer, edades 15-81 años) que se encontraban realizando la pesca al momento de la visita o que fueron referidas por otros pescadores.

Caracterización de la pesquería artesanal, perfil del pescador y amenazas

Se diseñó un instrumento de tipo mixto que, por su factibilidad de aplicación en campo, fue empleado como encuesta y guía de entrevista, llenado por el entrevistador-encuestador (Rojas-Soriano, 2013). Esta adaptación se basó en lo sugerido por la FAO (2001) para la recopilación de datos relativos a la pesca, y por los trabajos de Errazti et al. (1998), De la Cruz-González et al. (2011) y Mercado-Silva et al. (2011).

La actividad pesquera se caracterizó de manera cualitativa a partir de 22 preguntas sobre las artes de pesca, embarcaciones, especies objetivo, capturas y esfuerzo pesquero, y con observación directa. La verificación de las especies objetivo se apoyó en imágenes de las especies de peces presentes en la región impresas en lonas de $0,5 \mathrm{~m}$ de alto y póster tamaño tabloide. Para caracterizar el perfil de los pescadores se incluyeron nueve preguntas sociodemográficas en el cuestionario y siete sobre su experiencia en la actividad (p.e., años de práctica y motivaciones principales).

Las amenazas al suministro de SC se determinaron considerando como indicador la percepción de los pescadores sobre los cambios que han ocurrido en la pesca y/o el entorno y sus causas, tomando como referencia el tiempo en que iniciaron esta actividad y el presente. La información se obtuvo a partir de tres preguntas y se analizó en función de la experiencia en años de los pescadores, clasificándolos sobre esa base como jóvenes ( $<16$ ańos, $n=12)$, maduros (18-42 años, $n=15)$ y veteranos (>43 años, n=19) (Sáenz-Arroyo et al., 2005). Las diferencias entre los cambios y sus causas se analizaron con la prueba Kruskal-Wallis (Kruskal \& Wallis, 1952) y comparaciones pareadas a posteriori mediante pruebas de bondad de ajuste Ji cuadrada $(\alpha=0,05$, g.l.=5 y 4$)$ en el software Minitab 17 (Minitab 17 Statistical Software, 2010).

Importancia de los servicios ecosistémicos culturales (sc)

En este estudio se analizaron seis sc (Tabla 1), determinados a partir del protocolo GRACE (Guidance for the rapid assessment of cultural ecosystem services), en Infield et al. (2016):

1. Relaciones sociales y comunidad (Rsyc)

2. Herencia cultural, sentido de pertenencia e identidad (HCeI)

3. Educación y conocimiento ecológico (EyCE) 


\section{Salud física y mental (sғум) \\ 5. Recreación y turismo (вут) \\ 6. Valores estéticos (VE)}

Su valoración directa se realizó con preguntas en escala Likert (Likert, 1932), asignando puntajes 1-5: totalmente en desacuerdo (1), en desacuerdo (2), neutral (3), de acuerdo (4), totalmente de acuerdo (5) (Rodrigues, 2015). Se consideró que el pescador identificó un sc cuando asignó puntajes de 4 y 5 . Cuatro sc se evaluaron mediante una pregunta (RSYC, EYCE, RYT Y VE), mientras que los servicios restantes se abordaron con tres, debido a su complejidad (HCer y sFym). La valoración de SC con más de una pregunta se estableció mediante la media aritmética.

Para complementar el entendimiento de los sc, se formularon preguntas suplementarias. Estas incluyeron aspectos sobre las motivaciones para realizar la pesca, la forma y edad en que se aprendió la actividad, así como el origen del conocimiento de los sitios en los que se lleva a cabo. Se interrogó también sobre la práctica de la actividad en el presente (uso de capturas y acompañamiento durante la realización de la actividad). Para reforzar la comprensión de la importancia de la actividad para el entrevistado, se incluyó una pregunta hipotética sobre su sentir en caso de no poder desempeńar la pesca en el futuro.

Las relaciones entre las preguntas suplementarias y sc (Figura 2) se analizaron mediante un análisis de correspondencias múltiples (АСм) (Le Roux \& Rouanet, 2010) en el software spss versión 20 С Івм Corp. La posible influencia de factores como la experiencia en el ejercicio de la pesca, procedencia, escolaridad, ocupación, parentesco con la persona que les enseñó a pescar y uso de los peces en la valoración de sc se determinó con pruebas de hipótesis Kruskal-Wallis (Kruskal \& Wallis, 1952). 


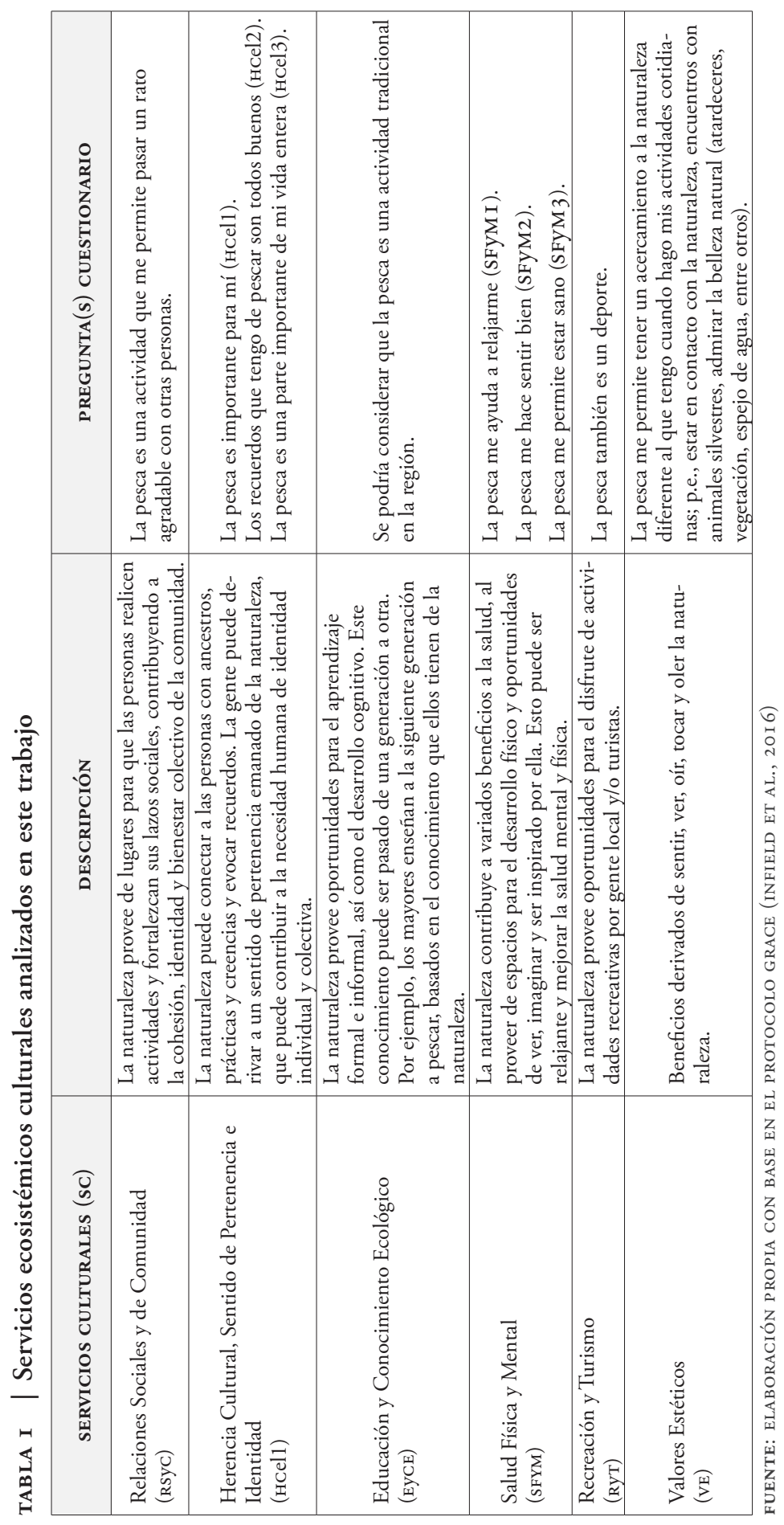


FIGURA 2 | Red conceptual de los sc analizados y sus relaciones con las preguntas suplementarias
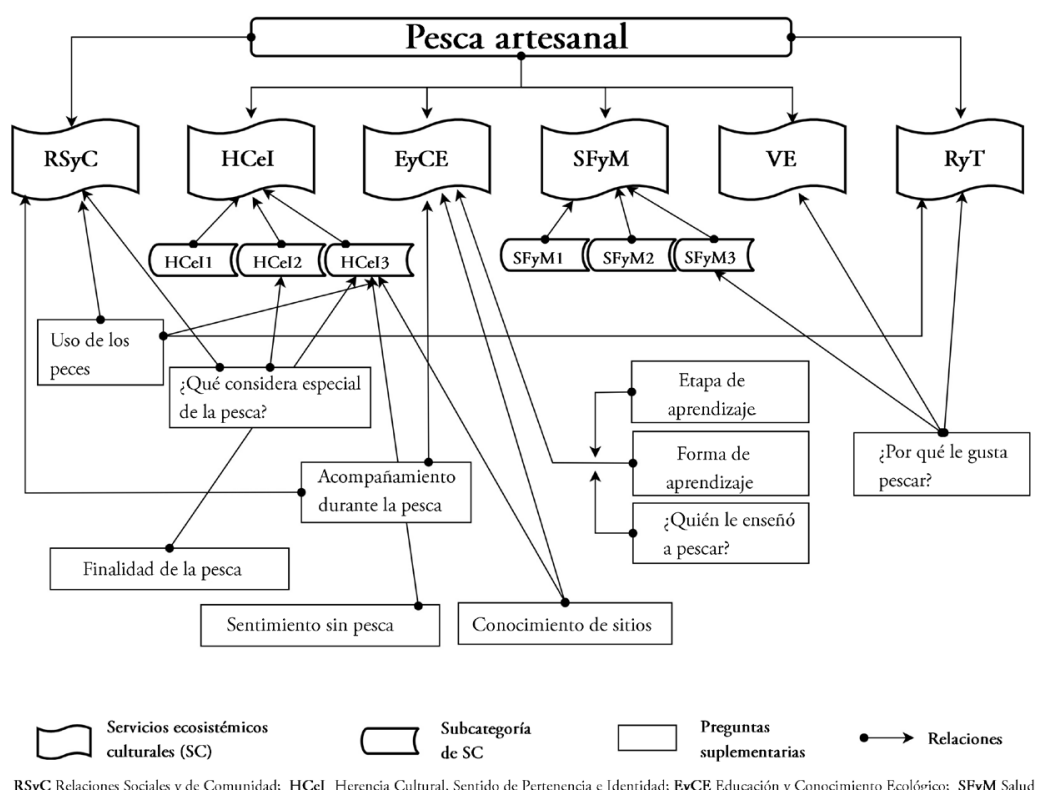

Física y Mental; RyT Recreación y Turismo; VE Valores Estéticos

FUENTE: ELABORACIÓN PROPIA

\section{Análisis espacial del flujo de los servicios ecosistémicos culturales (sc)}

El flujo o suministro de un servicio ecosistémico se refiere a la cantidad de sus beneficios que son recibidos por los usuarios en un área y tiempo específicos (Syrbe et al., 2017). Por lo tanto, en esta investigación, el flujo de sc ocurriría en los sitios donde se desarrollan las actividades pesqueras y los pescadores los identifican. Para ubicarlos, fueron empleadas técnicas de mapeo participativo (Raymond et al., 2009). Se usó como apoyo un mapa de la región generado en gran formato (1,5 m alto, lona), que incluía referencias a ríos principales y secundarios, cuerpos de agua, principales localidades, redes carreteras y de terracería. Los pescadores (o el entrevistador por solicitud de ellos) colocaron etiquetas redondas de colores en los sitios y zonas de pesca. Esta información se georreferenció posteriormente con el software Google Earth ${ }^{\circledast}$ y compiló en una base de datos espacial a la que se añadieron las valoraciones de los SC identificados por los usuarios.

El flujo de los sc se determinó con un análisis de Densidad Kernel (Nahuelhual et al., 2014), en el software ArcGis (Environmental Systems Research Institute [ESRI], 2012). A este análisis se le incorporó la valoración promedio de los sc otorgada por cada pescador. El flujo de los servicios se cuantificó de manera cualitativa en seis categorías: no identificado, ${ }^{5}$ muy bajo, bajo, medio, alto y muy alto, y se obtuvo la proporción de la red hidrológica correspondiente a cada una.

5 Esta categoría comprendió aquellas zonas donde el modelo de Densidad Kernel no proporcionó información. 


\section{Resultados}

En la subcuenca, 98\% de los pescadores son hombres, con edades entre 15 y 81 ańos (Figura 3A), oriundos de la región $(82,1 \%)$ o avecindados de residencia promedio 24 años (9,2\%). Cuentan con escolaridad básica (50\%) y se distribuyen en todos los sectores económicos. Aprenden la pesca de manera informal durante edades tempranas (niñez, 37\%; adolescencia, 39,1\%); son enseñados por familiares $(46,7 \%)$, amigos $(24,4 \%)$ y el $28,9 \%$ es autodidacta. La pesca es una actividad habitual en la región, desarrollada todo el ańo, con menor intensidad durante el periodo de lluvias (julio-octubre). La mayoría de los sujetos (50\%) pesca por lo menos una vez a la semana en compañía de familiares, amigos o ambos (Figura 3B), dedicando a ello de tres a seis horas.

FIGURA 3 | Características de la pesquería artesanal estudiada
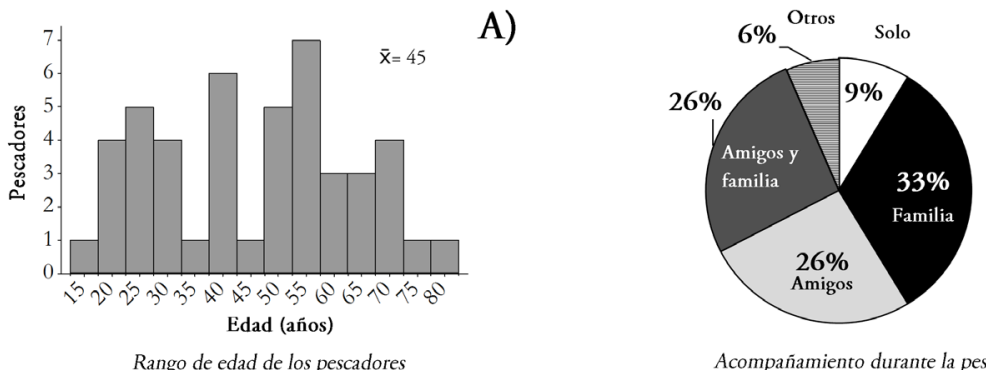

B)

A)

Acompañamiento durante la pesca

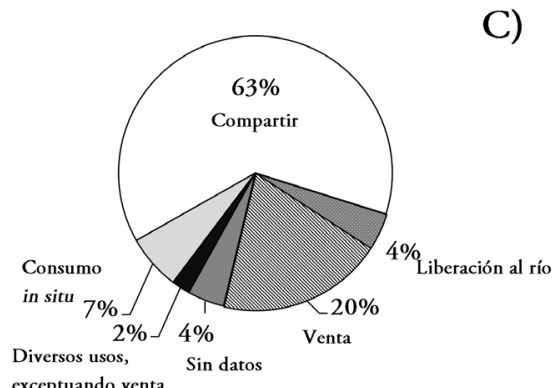

Uso de los peces

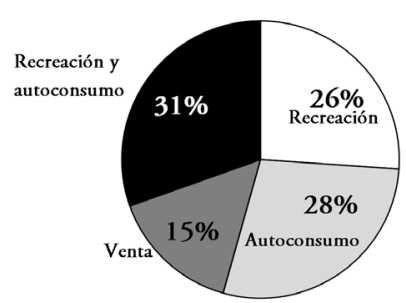

Motivaciones por las que pesca

D)

Nota: A) EdAd de los pescadores, B) acompañamiento duRANTe Las aCtividades de pesca, C) USO DE LOS PECES, Y D) MOTIVACIONES PARA PESCAR.

FUENTE: ELABORACIÓN PROPIA

En ríos de la parte alta se pesca principalmente con artes mixtas: atarraya y anzuelos (conocidas localmente como tarraya y piolas, respectivamente), mientras que los pescadores de la parte baja emplean mayor diversidad de artes de pesca: trampas, nasas (construidas con vegetación nativa de la zona), trasmallos y líneas de anzuelo (Figura 4). La pesca en ambientes lénticos ocurre principalmente en la Presa 
Corrinchis e involucra el uso de pequeñas embarcaciones: kayak, canoas y embarcaciones menores de remos (37\% de los entrevistados refirió utilizarlas y 22\% de ellos posee algún tipo de embarcación). La pesca en este embalse es la única regulada y de acuerdo con la Norma Oficial Mexicana NOM-o6o SAG/PESC-20 I 4 (Diario Oficial de la Federación [DOF], 2014), y está restringida a realizarse desde la orilla, con anzuelo o cańa y mediante permiso de pesca deportiva. El uso de embarcaciones y trasmallos (tumbos) para pesca comercial está autorizado solo a miembros de la única cooperativa de pescadores de la región: PROFERCo. Es importante señalar que esta se fundó por habitantes de las localidades afectadas por la construcción de la presa -La Providencia, San Fernando y Corrinchis-, quienes relataron haber cambiado su medio de vida de la agricultura por la pesca al verse imposibilitados de desempeñar la actividad debido a la inundación de sus tierras.

FIgura 4 | Pescadores artesanales de la subcuenca hidrológica del río Mascota

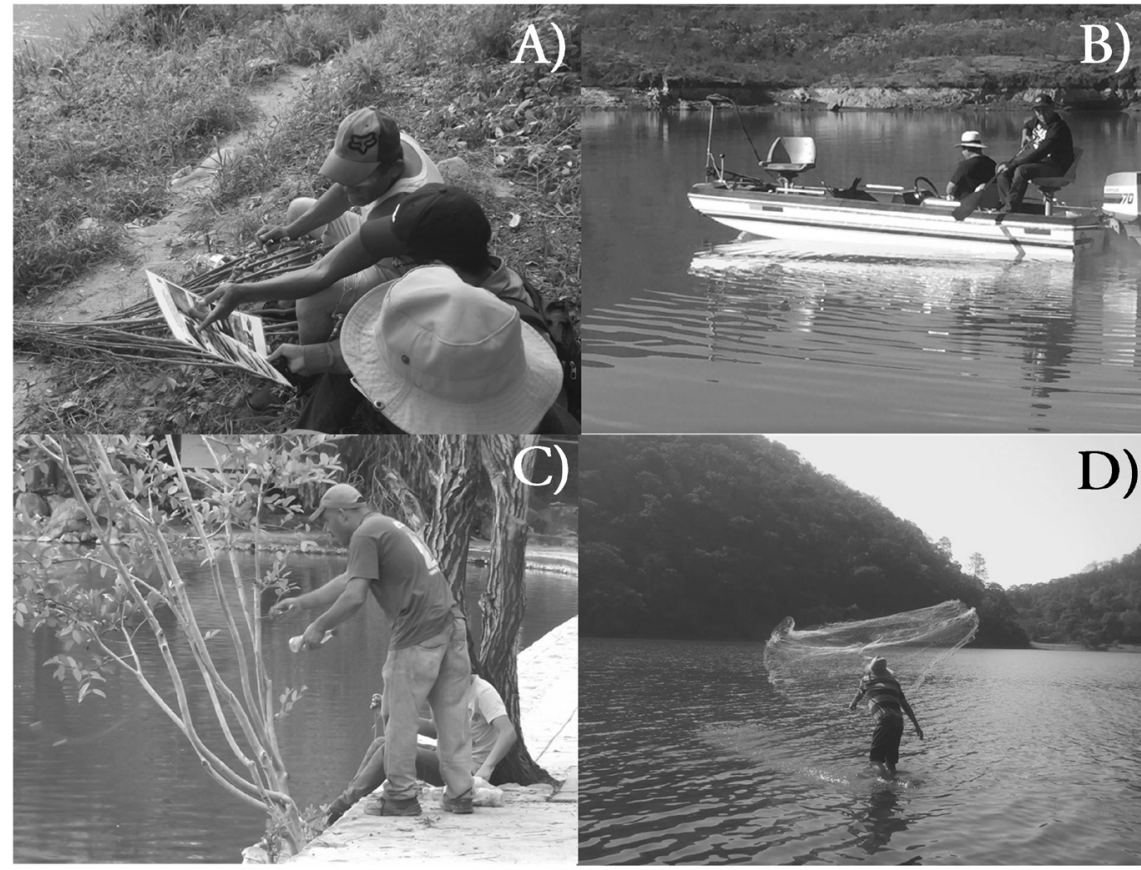

NOTA: A) PESCADOR DE LA DESEMBOCADA CONSTRUYENDO UNA NASA MIENTRAS IDENTIFICA LAS ESPECIES OBJETIVO DE LA PESCA DURANTE LA ENTREVISTA; B) PESCADORES DEPORTIVOS PARTICIPANDO EN TORNEO DE PESCA EN LA PRESA CORRINCHIS; C) PADRE E HIJO PESCANDO CON PIOLA EN LA LAgUNA DE YERBABUENA; D) PESCADOR EMPLEANDO ATARRAYA, LAGUNA DE JUANACATLÁN.

FUENTE: A, C-D) PRIMERA AUTORA, ERÉNDIRA CANALES-GÓMEZ; B) GOBIERNO DE MASCOTA

Las especies objetivo en la parte media de la subcuenca (Talpa y Mascota) son de origen introducido: tilapia (Oreochromis niloticus), carpa (Cyprinus carpio) y lobina (Micropterus salmoides), aunque existe pesca en menor grado de las especies nativas 
boquinete (Moxostoma austrinum) y bagre (Ictalurus dugesii). En la parte baja se pescan especies nativas: langostino (Macrobrachium sp.), cristalina (Gobiomorus maculatus), guavina (Eleotris picta), trucha de río (Dajaus monticola) y la especie introducida "mojarra" (tilapia). La captura se usa para autoconsumo y regalo a familiares y amigos como muestra de afecto (69,6\%). Solo 19,6\% refirió venderla. Los volúmenes de captura son variables y bajos, oscilan entre 1 y $10 \mathrm{~kg}$ (autoconsumo) y reportando hasta $40 \mathrm{~kg}$ (venta) por pescador. El 86,9\% de ellos disfruta la actividad por los beneficios no lucrativos que proporciona: recreación, alimentos y contacto con la naturaleza, siendo concordante con las principales motivaciones identificadas (Figura 3D). La pesca es una actividad importante para los entrevistados: al responder sobre qué sentirían si ya no pudieran realizarla, $79 \%$ aludió a sentimientos negativos, variando desde malestar general (48\%), hasta mencionar que una parte de ellos les faltaría (Figura 5A). Como nos señalaron estos dos pescadores:

Sería como si una parte de tu todo se perdiera. (28 años)

Se acaba una tercera parte de mí, se apaga uno. (29 años)

Estos afectos fueron más representativos de quienes aprendieron en etapas tempranas, mientras que la proporción de pescadores indiferentes o que buscarían otro pasatiempo fue mayor en aquellos que lo hicieron durante la juventud y adultez (Figura 5B).

FIGURA 5 | Porcentaje de respuestas (A) y categorizado por etapa de aprendizaje (B) a la pregunta ¿Cómo se sentiría usted si ya no pudiera realizar más la pesca porque los peces se terminaran o el río estuviera muy contaminado?
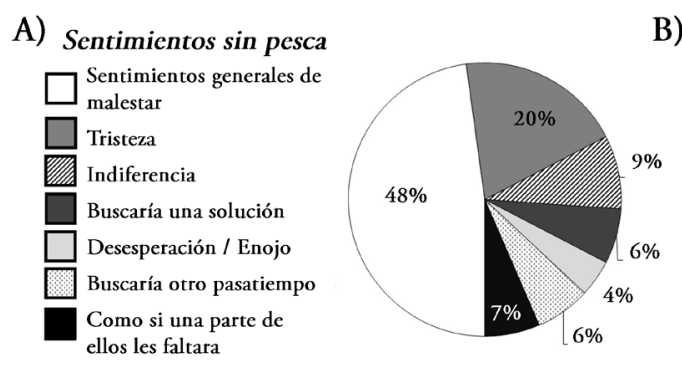

B)

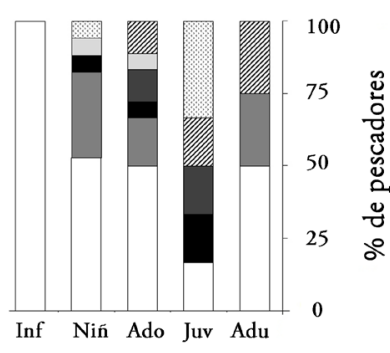

Etapa de aprendizaje

Inf= infancia; Niń $=$ Nińez; Ado $=$ adolescencia; Juv $=$ juventud; Adu $=$ Adulte

FUENTE: ELABORACIÓN PROPIA

Importancia de los servicios ecosistémicos culturales (sc)

Los pescadores identificaron todos los sc con una valoración promedio $>4$, no mostrando diferencias significativas entre pescadores con diferente experiencia, procedencia y escolaridad $(p>0,05)$. Sin embargo, sí se observó una valoración diferenciada en el servicio sғyм [Salud Física y Mental] ( 1 y 2 ) de acuerdo con la 
ocupación, no siendo identificado por la única mujer entrevistada, que se dedica al hogar $(p=0,04$ y $p=0,038)$. Los autodidactas no identificaron los servicios Hcel1 [Herencia Cultural, Sentido de Pertenencia e Identidad] у вут [Recreación y Turismo] $\left(\overline{\mathrm{X}}_{\mathrm{HCel1}}=3,85 ; \overline{\mathrm{X}}_{\mathrm{RyT}}=3,54\right)$ a diferencia de aquellos que aprendieron por un familiar o amigo $\left(\overline{\mathrm{X}}_{\mathrm{HCel} 1}=4,49 ; \overline{\mathrm{X}}_{\mathrm{RyT}}=4,65\right)(p=0,046)$. Los pescadores deportivos no reconocieron el servicio Rsyc $(p>0,01)$. Por su parte, quienes usan los peces para venta no identificaron el SC RyT $\left(\overline{\mathrm{X}}_{\mathrm{RyT}}=3,56 ; p=0,013\right)$, que recibió la valoración máxima por los deportivos.

FIGURA 6 | Análisis de correspondencias múltiples mostrando las relaciones entre los sc y variables suplementarias identificadas por los pescadores de la subcuenca del río Mascota

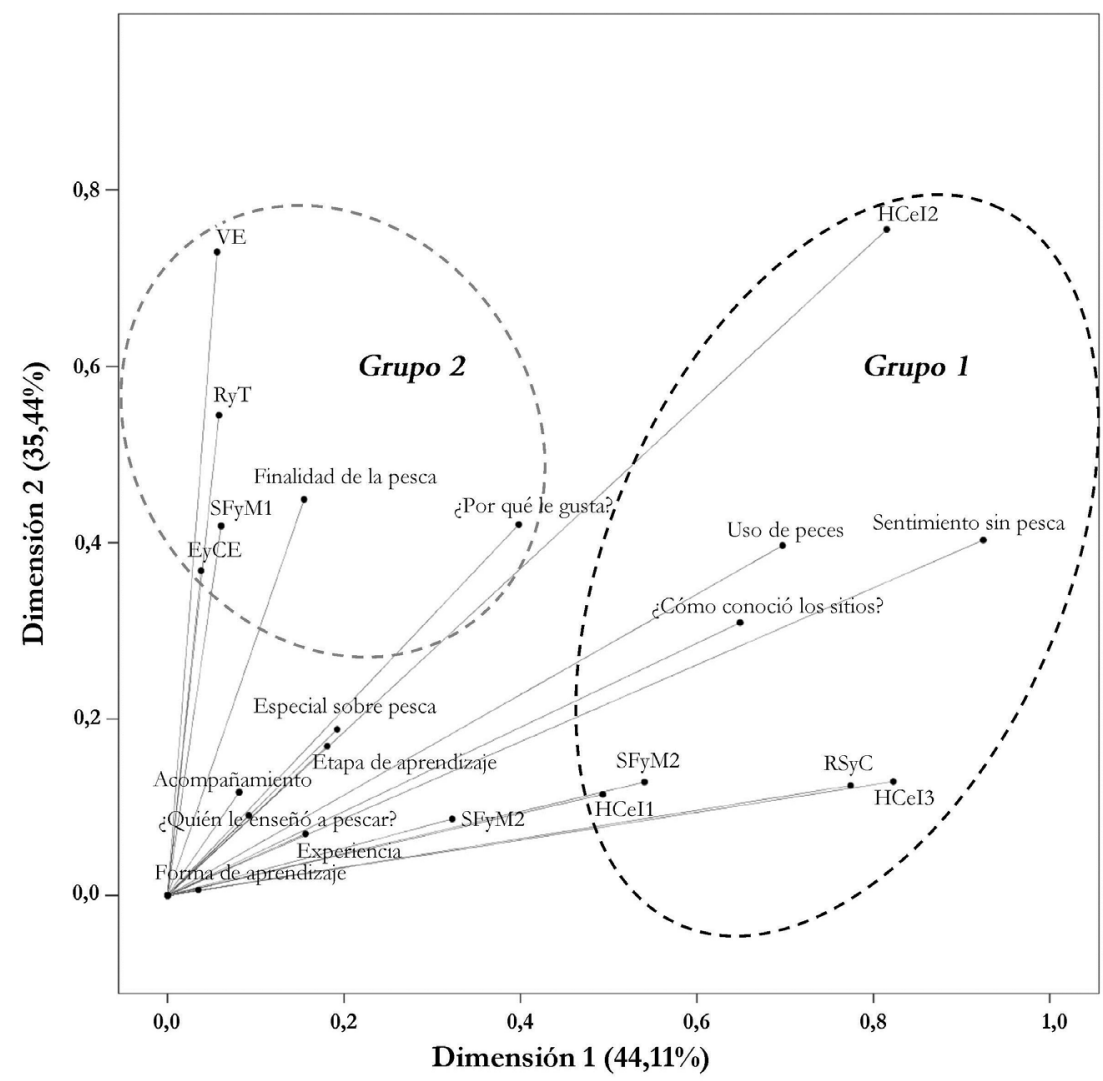

RSyC Relaciones Sociales y de Comunidad; HCeI Herencia Cultural, Sentido de Pertenencia e Identidad; EyCE Educación y Conocimiento Ecológico; SFyM Salud Física y Mental; RyT Recreación y Turismo; VE Valores Estéticos

NOTA: ALFA DE CRONBACH: DIMENSIÓN I= 0,9I 6; DIMENSIÓN 2=0,878.

FUENTE: ELABORACIÓN PROPIA 
El análisis de correspondencias múltiples mostró la existencia de dos grupos de sc (Figura 6). En el primero se asociaron las variables de sentimiento sin pesca, uso de los peces y conocimiento de los sitios de pesca, las cuales están agrupadas dentro de los servicios culturales de HCeI y Rsyc (Figura 2). Este grupo fue catalogado como Comunitario, debido a que está relacionado a valores colectivos y lazos sociales. En el segundo se relacionaron las variables de finalidad de la pesca y por qué les gusta pescar, que se agrupan en los servicios ecosistémicos culturales ve [Valores Estéticos], Rут [Recreación y Turismo], sFyм2 [Salud Física y Mental 2] y eyce [Educación y Conocimiento Ecológico]. A este último grupo se le denominó Individual, debido a que estos beneficios resultan de la interacción personal con la actividad.

\section{Flujo de servicios ecosistémicos culturales (sc)}

Los pescadores identificaron 100 sitios y 16 zonas donde reciben los sc. El conocimiento de estos lugares se transmite a través de familiares $(28,3 \%)$ o terceros (amistades y mayores, 26,1\%); otros son espacios del dominio popular o conocidos por la cercanía al domicilio del pescador $(21,7 \%)$, y el $11 \%$ de los entrevistados refirió descubrirlos por sí mismos.

FIGURA 7 | Distribución espacial del flujo de servicios ecosistémicos culturales en el área de estudio

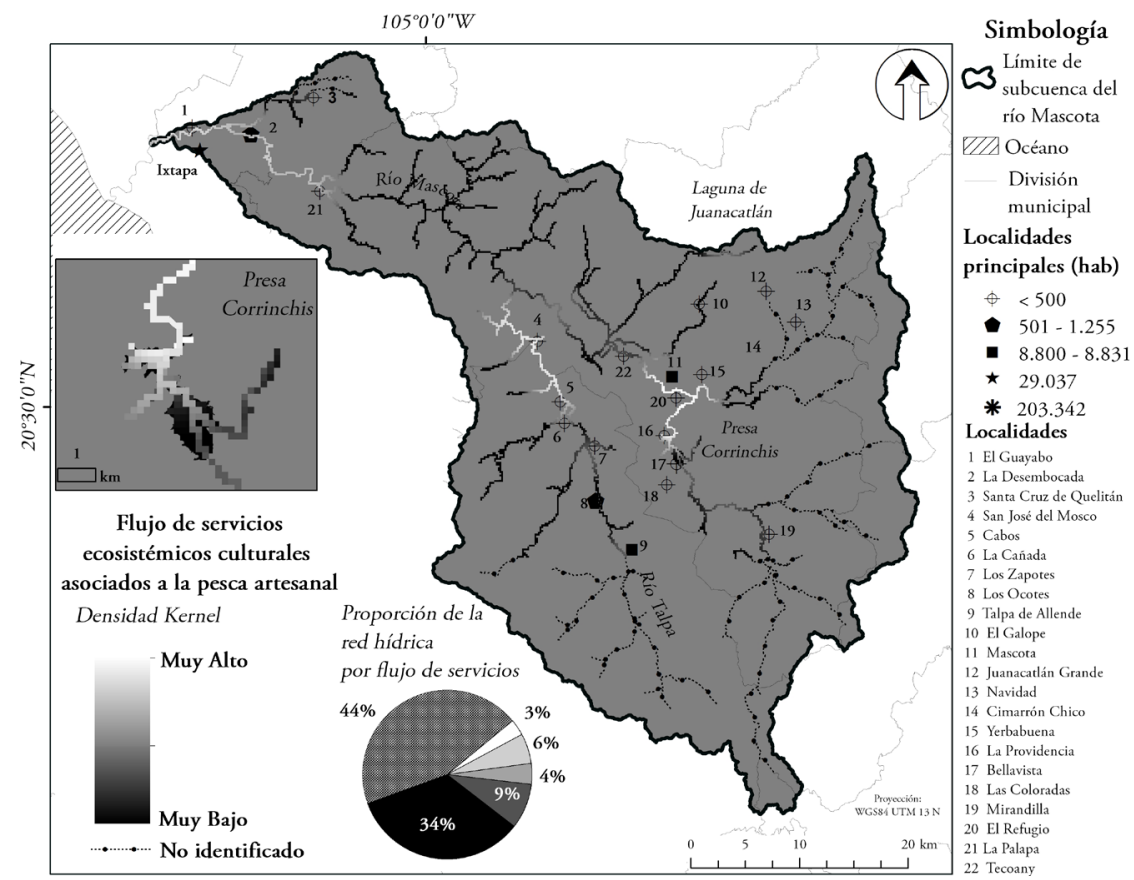

FUENTE: ELABORACIÓN PROPIA 
El análisis Kernel estimó que el flujo de sc ocurre en 66\% de la red hidrológica y cuerpos de agua, de los cuales $13 \%$ registró categorías de medio a muy alto. Se identificaron tres zonas principales de flujo de sc: i) Presa Corrinchis-río Mascota, ii) Desembocadura río Mascota-La Palapa, y iii) San José del Mosco-Los Zapotes (Figura 7). Los ríos de las partes altas de la subcuenca, antes de los poblados de Talpa, Mirandillas y Cimarrón Chico, no fueron identificados como sitios de pesca.

\section{Amenazas de los servicios ecosistémicos culturales (sc)}

El $98 \%$ de los pescadores notó cambios en su entorno o en la pesca durante el tiempo que lleva desarrollando la actividad, principalmente disminución en las capturas y menor abundancia de peces (61\%) (Figura 8A). Casi en su totalidad, las causas identificadas fueron de origen antrópico (Figura 8B). Las principales estuvieron asociadas al desarrollo de la pesca (A2, 28\%): sobreexplotación, malas prácticas (empleo de venenos y pesca de organismos juveniles) y falta de siembra de organismos en la Presa Corrinchis. Otras amenazas importantes fueron modificaciones a los sistemas naturales (A3, 26\%), principalmente la pérdida del bosque de ribera y la alteración de la dinámica y morfología de los ríos (Figura 9). Algunas respuestas de los entrevistados al respecto fueron:

Cada año destruyen el río; antes había sombra, sauces, jarales; cada ańo acaban con el río, cada año se va muriendo la naturaleza. (Pescador, 34 años)

Escarban en el río y acaban con el hábitat, hay menos árboles. (Pescador, 55 años) Los peces disminuyeron cuando tumbaron los árboles para levantar para que no se desbordara el río. (Pescador, 50 ańos)

FIgURA 8 | Cambios identificados por los pescadores artesanales de la subcuenca del río Mascota (A y C) y sus causas (B y D)

A)

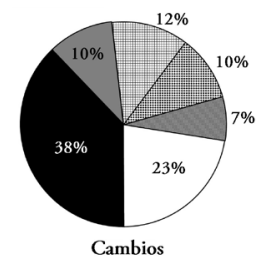

$\square 1 \square 2 \square 3$ 困 $\square^{2} \square^{5} \square$

C)

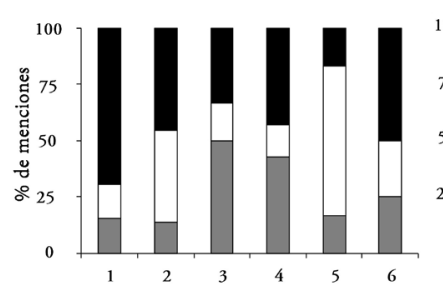

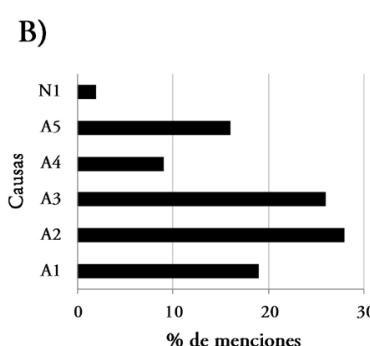

D)

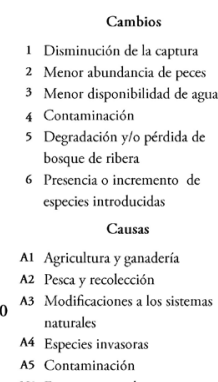

As Contaminación
N1 Factores naturales

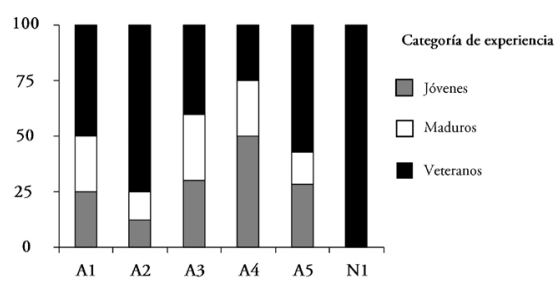

NOTA: LAS FIgURAS A Y B MUESTRAN LOS PORCENTAJES TOTALES, MiENTRAS QUE LA C Y D LO HACEN POR CATEGORÍA DE EXPERIENCIA.

FUENTE: ELABORACIÓN PROPIA 
El vertido de desechos agrícolas y el arrastre hacia los ríos de cenizas derivadas de la quema de coamiles por actividades agrícolas y ganaderas (A1), representaron 19\% de las menciones. La contaminación, principalmente por aguas negras y residuos sólidos (A5, 16\%), y presencia y efectos de especies introducidas (A4, 9\%), también se mencionaron como causas de cambios. Solo el $2 \%$ de las menciones se relacionó con procesos naturales; en específico, el cambio climático.

FIGURA 9 | Modificaciones a los sistemas naturales en dos sitios con muy alto flujo de sc de la localidad de Mascota

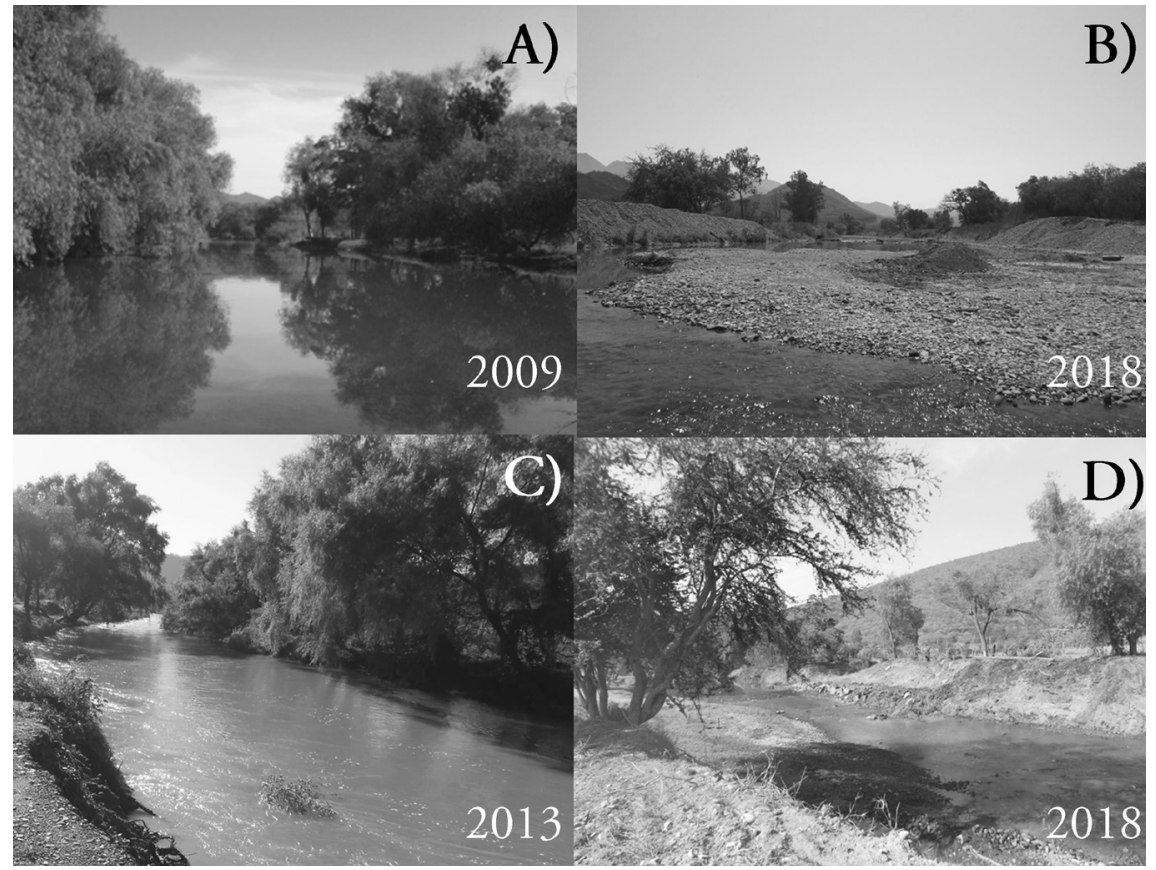

NOTA: A) Y B) JUNTO AL VADO, AÑOS 2009 Y 20 I 8; C) Y D) FRENTE AL PARQUE ALAMEDA 2, AÑOS $20 I 3$ Y 2OI8. SE PUEDE OBSERVAR LA PÉRDIDA DEL BOSQUE DE RIBERA Y LA MODIFICACIÓN DEL CAUCE COMO CONSECUENCIA DEL DRAGADO Y CONSTRUCCIÓN DE UN PUENTE Y BORDOS

FUENTE: A) HTTPS://BIT.LY/2VM3HU8; B-D) PRIMERA AUTORA, ERÉNDIRA CANALES-GÓMEZ

Los pescadores más experimentados identificaron cambios observables a largo plazo (disminución de capturas y de abundancia de peces, presencia de especies invasoras), mientras que los jóvenes mencionaron afectaciones más recientes (merma en la disponibilidad de agua, contaminación) $(\mathrm{p}=0,00)$ (Figura $8 \mathrm{C}$ ). Los maduros notaron en mayor proporción la degradación y/o pérdida del bosque de ribera $(\mathrm{p}=0,00)$. Los factores referidos fueron similares entre jóvenes y maduros, principalmente los efectos de especies introducidas y alteraciones a los sistemas naturales (Figura 8D) $(\mathrm{p}=0,334)$. Por su parte, los veteranos consideraron como principales causas las malas prácticas pesqueras y efectos derivados de la agricultura y ganadería $(\mathrm{p}=0,00)$. 


\section{Discusión}

Los SC presentan un alto valor social en la subcuenca del río Mascota, debido a que constituyen elementos fundamentales de identidad colectiva e individual de los pescadores. Se identificó que esta actividad es un componente del modo de vida de los pobladores de la subcuenca y fuente de otros servicios ecosistémicos, además del suministro de alimentos y recreación, con los que comúnmente se asocia. Estos argumentos coinciden con lo señalado por Chan et al. (2012) y Oleson et al. (2015), quienes han encontrado que los valores inmateriales asociados con la pesca pueden importar más a los pescadores que los beneficios materiales (dinero y cambios físicos deseables, como el sustento o la vivienda). Estas relaciones son descritas por Jiménez de Madariaga y García del Hoyo (2014) por medio del concepto "cultura de la pesca", que comprende, entre otras, las maneras de relacionarse y entender la vida, habitar el espacio, paisajes y comensalidad, además de la obtención de pescado para consumo.

Los pescadores aprenden la actividad de manera temprana mediante transmisión cultural del conocimiento ecológico tradicional de tipo vertical y horizontal (Cavalli-Sforza et al., 1982; Hewlett \& Cavalli-Sforza, 1986). Kals et al. (1999) han encontrado que las experiencias vividas durante la niñez y en compañía de familiares pueden derivar en el desarrollo de "afinidad emocional a la naturaleza", el mismo que se fortalece a medida que se realizan más contactos. Esto se observa en el tema "sentimiento sin pesca" y la frecuencia con la que se desarrolla la actividad. Tal afirmación se fortalece por las altas valoraciones otorgadas a los SC analizados, así como las relaciones en el análisis de correspondencias múltiples ( $\mathrm{ACM}$ ) entre los servicios HCer y Rsyc con las variables suplementarias. El acercamiento con la naturaleza es muy importante en la nińez, debido a que contribuye al desarrollo de conocimiento y valores relacionados con el medioambiente (Bögeholz, 2006). Sin embargo, se ha observado que la cantidad de niños carentes de experiencias al aire libre se ha incrementado, siendo la pesca la actividad más afectada (Floyd et al., 2006; Soga \& Gaston, 2016). Este fenómeno, denominado "extinción de la experiencia” (Pyle, 1993; Soga \& Gaston, 2016), puede generar cambios emocionales, actitudinales, de salud y de comportamiento hacia la naturaleza, los cuales son factores que alteran el bienestar humano y la sustentabilidad. En este contexto, es prioritario que se continúe y se promueva el ejercicio de la pesca, principalmente a edades tempranas.

Además de la obtención de alimento, la pesca es una actividad atractiva por su componente de aventura y desafío (Pollnac \& Poggie, 2008) y por sus beneficios a la salud, como la relajación, reducción de estrés y de emociones negativas (Floyd et al., 2006; Lynch et al., 2016). Estos beneficios, y otros servicios ecosistémicos que proveen las pesquerías artesanales, están estrechamente vinculados con las tres dimensiones del bienestar humano: material, relacional y subjetiva, de acuerdo con Weeratunge et al. (2014) y Cooke et al. (2016). Tales vínculos se observaron en el presente estudio al identificar cómo los pescadores se benefician de la pesca mediante la obtención de alimento e ingresos económicos (material), el desarrollo de relaciones sociales y vínculos dentro de la comunidad (relacional), a lo que se suma la importancia de la actividad en la identidad personal y como forma de 
recreación (subjetiva). Sin embargo, tales beneficios no son recibidos y valorados de manera semejante por todos los involucrados, como se observó en el caso de los servicios del grupo comunitario no reconocidos por pescadores deportivos (RSyC) y autodidactas (Hcel1), o la nula importancia de RyT por los pescadores comerciales. Estas valoraciones diferenciadas son relevantes, ya que proporcionan información sobre los subgrupos de actores clave que integran la pesquería artesanal de la región, la forma en la que interactúan con la naturaleza y los resultados de estas interacciones, que son recibidos en forma de sc (Lau et al., 2018). En conjunto con el análisis espacial del flujo de estos sc, ellos representan un insumo para el análisis de la pesquería artesanal como un socioecosistema adaptativo complejo, según ha sido propuesto por Arlinghaus et al. (2017).

Las características generales de la actividad pesquera de la subcuenca del río Mascota, incluidos sus vínculos con la cultura, identidad, estilo de vida de los pescadores y escasa participación de las mujeres, corresponden a una típica pesquería artesanal de aguas interiores de países en desarrollo (Sharma, 2011). Con respecto al papel de la mujer en la pesca, en Latinoamérica se ha dado tradicionalmente en el procesamiento y venta de las capturas (Arce-Ibarra \& Charles, 2008; Perea-Bláquez \& Flores Palacios, 2016; World Bank, FAO \& International Fund for Agricultural Development [IFAD], 2008). La brecha de género ha sido abordada por Floyd et al. (2006) en pesca recreativa, atribuyéndola a diversas causas; entre ellas, a las prioridades de las mujeres (familia y trabajo), la consideración de que ciertas formas de recreación no son apropiadas para ellas, o por preocupaciones en su seguridad para y durante su realización. Tales factores se reflejan en este estudio, donde solo se observó a una mujer efectuando la actividad. Es preciso notar que la participación de ellas en las pesquerías artesanales es opacada por sesgos metodológicos que llevan a omitir su trabajo en ese ámbito (Kleiber et al., 2014), por lo que es necesario que los estudios de pesquerías incorporen la perspectiva de género (FAO, 2018).

Otro aporte de este estudio fue el uso del conocimiento ecológico tradicional en el mapeo del flujo de sc, que permitió identificar las zonas de importancia para la comunidad y el suministro de los mismos. Este conocimiento ha sido utilizado previamente en el estudio de pesquerías artesanales (Schafer \& Reis, 2008); sin embargo, su empleo en combinación con los métodos de mapeo ha sido incipiente en la evaluación de sc (Nahuelhual et al., 2014; Schnegg et al., 2014), aunque tal enfoque ha probado ser una técnica adecuada para ello. El análisis espacial del flujo en conjunto con la identificación de las amenazas permitió advertir sobre la vulnerabilidad de los SC ante los procesos de expansión urbana y cambio de uso de suelo que experimenta la región debido a su cercanía con las localidades principales. Además, la información precisa sobre la ubicación y extensión de las zonas de pesca permitió identificar el desplazamiento realizado por los pescadores para visitarlas, que en ocasiones involucra recorrer largas distancias a pie $(\sim 10 \mathrm{~km})$, cargando el peso de las artes de pesca y capturas. Este esfuerzo, en conjunto con las principales motivaciones identificadas y el uso de los peces, subraya la importancia de los SC para los pescadores.

Los métodos empleados también permitieron una aproximación de tipo línea base cambiante (Pauly, 1995), en donde se identificaron cualitativamente los cambios en 
la actividad pesquera que hicieron posible determinar las amenazas para el suministro de los sc. La menor abundancia de peces y disminución de las capturas, contaminación y pérdida del bosque de ribera, así como las causas antrópicas asociadas a ellos, coincidieron con las amenazas globales de las pesquerías continentales y sus contribuciones al bienestar humano (Beard et al., 2011; Hallwass et al., 2013; McIntyre et al., 2016) y fueron concordantes con lo reportado por Mercado-Silva et al. (2011) para la pesquería artesanal de la Reserva de la Biosfera de la Sierra de Manantlán al sur del estado de Jalisco. Este tipo de conocimiento ecológico tradicional corresponde a la esfera "vinculación de los ecosistemas" (Santha, 2008) y consiste en la noción de los pescadores sobre las relaciones que ocurren entre diferentes ecosistemas (por ejemplo, bosques, ríos, lagos y sistemas agrícolas) y los problemas que emergen de la ruptura o alteración de estos vínculos. Destaca como principal causa de los cambios identificados ciertas malas prácticas pesqueras ya mencionadas (sobreexplotación, empleo de venenos y pesca de organismos juveniles). Aunado a lo anterior, es fundamental considerar que la persistencia de estas acciones contrarias al manejo sustentable de los recursos también tiene su origen en el conocimiento tradicional, aunque surge de su interacción con los sistemas de valores presentes actualmente en la comunidad, como lo ha sugerido Santha (2008). Por lo tanto, es pertinente implementar estrategias de promoción y capacitación con los pescadores de la región sobre artes y métodos idóneos para contrarrestar la transferencia de malas prácticas y lograr una pesca sustentable, con base en el saber tradicional.

La segunda causa que representa una severa amenaza a la continuidad de los sc fue la modificación de los sistemas naturales, principalmente la degradación y pérdida del bosque de ribera. La importancia de estos ecosistemas en la provisión de diversos servicios (regulación de inundaciones, mantenimiento de hábitats para especies, purificación del agua y suministro de alimentos, entre otros) y mantenimiento de funciones ecosistémicas, ha sido bien documentada (Aylward et al., 2005; Celentano et al., 2017; Naiman et al., 2010). Se ha reportado que estos ambientes están implicados directamente en el manejo y conservación de las pesquerías continentales, debido a que son considerados como parte del hábitat de los peces (Naiman \& Latterell, 2005). Por otro lado, constituyen un espacio fundamental para la recreación y el suministro de otros sc además de los evaluados en este trabajo, como los relacionados a la inspiración y espiritualidad, los cuales son altamente dependientes de la integridad ecohidrológica de estos ambientes (Rodrigues, 2015). El flujo de los sc que ocurre en la zona riparia también se relaciona con su accesibilidad, vinculada a la tenencia de la tierra (Flores-Díaz et al., 2014) y a la longitud de estas zonas accesibles al público (Villamagna et al., 2014), que podría explicar el porcentaje de la red hidrológica de la subcuenca que no fue identificado como sitios de flujo de sc.

Las intervenciones de desarrollo - p.e., construcción de presas- que buscan incrementar la provisión de otros servicios ecosistémicos acoplados, como suministro de agua, producción de alimentos derivados de la agricultura y generación de energía hidroeléctrica (FAO, 2018; Lynch et al., 2019), generan compromisos o 
tradeoffs ${ }^{6}$ para los sc de las pesquerías continentales globales y el mantenimiento de la biodiversidad (Lau et al., 2018). Desencadenan una serie de impactos que se inician con cambios en la composición y abundancia de peces nativos, seguidos de introducción de especies exóticas (Agostinho et al., 2016) con la finalidad de recuperar o incentivar el servicio de suministro de alimentos y/o promover nuevos servicios y actividades económicas, como es el caso de la pesca recreativo-deportiva (FAO, 2018), tal y como se observó en este trabajo. Esto provoca cambios socioeconómicos en las localidades influenciadas por ellos (Alrajoula et al., 2016) y podrían derivar en conflictos sociales y pérdidas de sc y otros servicios, de no existir una adaptación a las nuevas condiciones, como la observada en los pescadores comerciales de la cooperativa PROFERCO. En este respecto, preocupa la dependencia actual de las pesquerías artesanales sobre especies no nativas (FAO, 2018). A pesar de su contribución en los servicios ecosistémicos mencionados, se debe puntualizar que estos beneficios son de corto tiempo, debido a que las especies no nativas pueden generar un efecto en cascada de pérdida de biodiversidad, degradación de los ecosistemas y sus servicios acoplados (Pope et al., 2016). Esta problemática supone un desafío para la recuperación de las comunidades nativas de peces, su revaloración como especies objetivo para el mantenimiento de las pesquerías artesanales y sus sc, particularmente en zonas con baja diversidad de especies como es la subcuenca del río Mascota, a diferencia de pesquerías multiespecíficas, como las del Amazonas en Brasil (Hallwass et al., 2013; Hallwass \& Silvano, 2016) o las localizadas en Colombia (Olaya-Rodríguez et al., 2017).

Finalmente, este trabajo pone en evidencia la importancia de incorporar los sc en el análisis de las pesquerías artesanales, dada su relevancia para la seguridad alimentaria, recreación y conservación de la biodiversidad acuática de países en desarrollo (FAO, 2018; Pomeroy, 2016). Atendiendo a la prioridad de incorporar estos hallazgos en los planes de desarrollo y ordenamiento (Lynch et al., 2019; McIntyre et al., 2016), se emiten las recomendaciones que a continuación se detallan y que pueden ser adaptadas a otros contextos similares, dada la afinidad de las características de la pesquería artesanal del río Mascota con otras de países en desarrollo (FAO, 2018; Sharma, 2011), y la factibilidad de replicar la metodología propuesta:

a. Promover buenas prácticas pesqueras con base en el saber tradicional y fomentar el desarrollo de esta actividad en edades tempranas.

b. Mejorar las prácticas agropecuarias y de tratamiento de aguas residuales para minimizar sus impactos en el ecosistema acuático y el suministro de servicios ecosistémicos.

c. Ejercer acciones de restauración y conservación de los bosques de ribera, particularmente en las zonas de mayor flujo de sc y garantizar que la población pueda tener libre acceso a estos ambientes.

6 De acuerdo con King et al. (2015), los compromisos o tradeoffs son el resultado del uso de suelo o decisión de manejo que conduce a incrementar un servicio ecosistémico y resulta en el decremento de otro(s) servicio(s). 
d. Incentivar la producción acuícola de especies nativas y realizar estudios de la dinámica ecohidrológica regional, que permitan orientar acciones de restauración en los cauces que favorezcan la recuperación y/o reintroducción de estas especies.

e. Ampliar los análisis de sc de las pesquerías, incluyendo otros servicios acoplados y actores sociales (Lau et al., 2018; Small et al., 2017).

\section{Conclusiones}

La pesca artesanal es considerada como una actividad tradicional, con transmisión de conocimiento ecológico de tipo vertical y horizontal. Mediante ella, los pescadores reciben los Sc Herencia Cultural e Identidad (HCer) y Relaciones Sociales y Comunidad (RSyc), que son esenciales para su vida e identidad colectiva; y los SC Salud Física y Mental (sғyм), Recreación y Turismo (Rут), Valores Estéticos (ve), y Educación y Conocimiento Ecológico (EyCE), importantes para su bienestar individual. Para asegurar la continuidad de la pesca y flujo de sc, es prioritario que los planes de desarrollo regionales implementen estrategias para su protección y el libre acceso, principalmente en las zonas de mayor flujo.

\section{Agradecimientos}

Este trabajo fue financiado por los proyectos 1842498/1842495, 1842517 de la División de Ciencias Biológicas y de la Salud, Centro Universitario de la Costa, Universidad de Guadalajara (Cucosta-Udeg). Eréndira Canales-Gómez agradece al Consejo Nacional de Ciencia y Tecnología (CONACYT) por la beca doctoral 291137 otorgada. Romina Díaz Gómez agradece al Consejo Nacional de Investigaciones Científicas y Técnicas (CONICET) por la beca postdoctoral otorgada. Nuestra gratitud a Hiromi Cuenca, Alicia Gómez, Diego Landín, Mauricio Regalado y Alfredo Canales por su apoyo en campo. Reconocemos a la Sociedad Cooperativa PROFERCo s.C.L. y las personas que accedieron a ser entrevistadas; así como las facilidades otorgadas por las Direcciones de Ecología de los Ayuntamientos de Mascota y Talpa de Allende, Jalisco 2015-2018. Gracias a Karen Peña por sus valiosos comentarios al manuscrito.

\section{Referencias bibliográficas}

Agostinho, A. A., Gomes, L. C., Santos, N. C. L., Ortega, J. C. G. \& Pelicice, F. M. (2016). Fish assemblages in neotropical reservoirs: Colonization patterns, impacts and management. Fisheries Research, 173, 26-36. https://doi.org/10.1016/j.fishres.2015.04.006

Alrajoula, M. T., Al Zayed, I. S., Elagib, N. A. \& Hamdi, M. R. (2016). Hydrological, socioeconomic and reservoir alterations of Er Roseires dam in Sudan. Science of The Total Environment, 566-567, 938-948. https://doi.org/10.1016/j.scitotenv.2016.05.029 
Arce-Ibarra, A. M. \& Charles, A. T. (2008). Inland fisheries of the Mayan zone in Quintana Roo, Mexico: Using a combined approach to fishery assessment for data-sparse fisheries. Fisheries Research, 91, 151-159. https://doi.org/10.1016/j.fishres.2007.11.015

Arlinghaus, R., Alós, J., Beardmore, B., Daedlow, K., Dorow, M., Fujitani, M., Hühn, D., Haider, W., Hunt, L. M., Johnson, B. M., Johnston, F., Klefoth, T., Matsumura, S., Monk, C., Pagel, T., Post, J. R., Rapp, T., Riepe, C., Ward, H. \& Wolter, C. (2017). Understanding and managing freshwater recreational fisheries as complex adaptive social-ecological systems. Reviews in Fisheries Science \& Aquaculture, 25(1), 1-41. https://doi.org/10.1080/23308249.2016.1209160

Ash, N., Bennett, K., Reid, W., Irwin, F., Ranganathan, J., Scholes, R., Tomich, T. P., Brown, C., Gitay, H., Raudsepp-Hearne, C. \& Lee, M. (2010). Assessing ecosystems, ecosystem services, and human well-being. En N. Ash, H. Blanco, C. Brown, K. García, C. Ruadsepp-Hearne, D. R. Simpson, R. Scholes, T. P. Tomich, B. Vira \& M. Zurek (Eds.), Ecosystems and human well-being. A manual for assessment practitioners (pp. 1-32). Island Press. http://www.ecosystemassessments.net/resources/ecosystemsand-human-well-being-a-manual-for-assessment-practitioners.pdf

Auerbach, C. F. \& Silverstein, L. B. (2003). Qualitative data: An introduction to coding and analysis. New York University Press.

Aylward, B., Bandyopadhyay, J., Belausteguigotia, J. C., Börkey, P., Cassar, A. Z., Meadors, L., Saade, L., Siebentritt, M., Stein, R., Tognetti, S. \& Tortajada, C. (2005). Freshwater ecosystem services. En K. Chopra, R. Leemans, P. Kumar \& H. Simons (Eds.), Ecosystems and human well-being: Policy responses (pp. 213-255). Island Press. https:// www.millenniumassessment.org/documents/document.312.aspx.pdf

Balvanera, P., Cotler, H., Aburto Oropeza, O., Aguilar Contreras, A., Aguilera Peña, M., Aluja, M., Andrade Cetto, A., Arroyo Quiroz, I., Ashworth, L., Astier, M., Ávila, P., Bitrán Bitrán, D., Camargo, T., Campo, J., Cárdenas González, B., Casas, A., Díaz-Fleischer, F., Etchevers, J. D., Ghillardi, A. (...), Zarco, A. (2009). Estado y tendencias de los servicios ecosistémicos. En Capital natural de México, vol. ii: Estado de conservación y tendencias de cambio (pp. 185-245). México: Comisión Nacional para el Conocimiento y Uso de la Biodiversidad (CONABIo). https://bioteca.biodiversidad.gob.mx/janium/ Documentos/7404.pdf

Baños Francia, J. A. (2018, septiembre 5-7). Resistencia institucional para implementar la Nueva Agenda Urbana en Puerto Vallarta, México. Ponencia presentada en CTv 2018: XII Congreso Internacional Ciudad y Territorio Virtual, uncuyo, Mendoza. https:// upcommons.upc.edu/handle/2117/132256

Beard, D. T., Arlinghaus, R., Cooke, S. J., McIntyre, P. B., De Silva, S., Bartley, D. \& Cowx, I. G. (2011). Ecosystem approach to inland fisheries: Research needs and implementation strategies. Biology Letters, 7(4), 481-483. https://doi.org/10.1098/rsbl.2011.0046

Berkes, F. (1999). Sacred Ecology. Traditional ecological knowledge and resource management. Taylor and Francis.

Bögeholz, S. (2006). Nature experience and its importance for environmental knowledge, values and action: recent German empirical contributions. Environmental Education Research, 12(1), 65-84. https://doi.org/10.1080/13504620500526529 
Cavalli-Sforza, L. L., Feldman, M. W., Chen, K. H. \& Dornbusch, S. M. (1982). Theory and observation in cultural transmission. Science, 218(4567), 19. https://doi.org/10.1126/ science.7123211

Celentano, D., Rousseau, G. X., Engel, V. L., Zelarayan, M., Oliveira, E. C., Araujo, A. C. M. \& de Moura, E. G. (2017). Degradation of riparian forest affects soil properties and ecosystem services in eastern Amazon of Brazil. Land Degradation \& Development, 28(2), 482-493. https://doi.org/ 10.1002/ldr.2547

Chan, K. M. A., Guerry, A. D., Balvanera, P., Klain, S., Satterfield, T., Basurto, X., Bostrom, A., Chuenpagdee, R., Gould, R. K., Halpern, B. S., Hannahs, N., Levine, J., Norton, B., Ruckelshaus, M., Russell, R., Tam, J. \& Woodside, U. (2012). Where are cultural and social in ecosystem services? A framework for constructive engagement. BioScience, 62(8), 744-756. https://doi.org/10.1525/bio.2012.62.8.7

Cooke, S. J., Allison, E. H., Beard, D. T., Arlinghaus, R., Arthington, A., Bartley, D., Cowx, I. G., Fuentevilla, C., Leonard, N. J., Lorenzen, K., Lynch, A. J., Nguyen, V. M., Youn, S.-J., Taylor, W. W. \& Welcomme, R. (2016). On the sustainability of inland fisheries: Finding a future for the forgotten. Ambio, 45, 753-764. https://doi.org/10.1007/ s13280-016-0787-4

De la Cruz-González, F. J., Morales-Pacheco, O., Ramos-Cruz, S. \& Luna-Raya, M. C. (2011). Perfil sociodemográfico y económico de los pescadores de las localidades del sistema lagunar La Joya Buenavista-Cordón Estuárico, Chiapas. Ciencia Pesquera, 19(1), 61-69.

Diario Oficial de la Federación [Dof ], México. (2014, mayo 27). Norma Oficial Mexicana NOM060-SAG/PESC-2014, Pesca responsable en cuerpos de aguas continentales dulceacuicolas de jurisdicción federal de los Estados Unidos Mexicanos. Especificaciones para el aprovechamiento de los recursos pesqueros. https://www.dof.gob.mx/nota_detalle.php?co digo $=5346129 \& f e c h a=27 / 05 / 2014$

Díaz, S., Demissew, S., Carabias, J., Joly, C., Lonsdale, M., Ash, N., Larigauderie, A., Adhikari, J. R., Arico, S., Báldi, A., Bartuska, A., Baste, I. A., Bilgin, A., Brondizio, E., Chan, K. M. A., Figueroa, V. E., Duraiappah, A., Fischer, M., Hill, R. (...), Zlatanova, D. (2015). The IPBES conceptual framework - Connecting nature and people. Current Opinion in Environmental Sustainability, 14, 1-16. https://doi.org/10.1016/j. cosust.2014.11.002

Dudgeon, R. C. \& Berkes, F. (2003). Local understanding of the land: Traditional ecological knowledge and indigenous knowledge. En H. Selin (Ed.), Nature Across Cultures: Views of Nature ami the Environment in Non- Western Culture (pp. 75-96). Khllver Academic Publishers.

Environmental Systems Research Institute [ESRI]. (2012). ARCGIS Release 10.1. Redlands, CA.

Errazti, E., Bertolotti, M. I. \& Pagani, A. (1998). Indicadores para el análisis socioeconómico de la actividad pesquera: Un estudio socioeconómico del pescador costero de la región bonaerense. Frente Maritimo, 17, 71-80. http://nulan.mdp.edu.ar/1349/

FAO [Food and Agricultural Organization]. (2001). Directrices para la recopilación sistemática de datos relativos a la pesca de captura. Documento Técnico de Pesca 382. http://www.fao. org/3/x2465s/x2465s00.htm

FAO [Food and Agricultural Organization]. (2016). The state of world fisheries and aquaculture 2016. Contributing to food security and nutrition for all. http://www.fao.org/3/ai5555e.pdf 
FAO [Food and Agricultural Organization]. (2018). Review of the state of the world fishery resources: Inland fisheries. FAO Fisheries Circular. N. ${ }^{\circ}$ C942 Revision3. http://www.fao. org/3/ca0388en/CA0388EN.pdf

Felipe-Lucia, M. R., Comin, F. A. \& Escalera-Reyes, J. (2015). A framework for the social valuation of ecosystem services. Ambio, 44(4), 308-318. https://doi.org/10.1007/ s13280-014-0555-2

Flores-Díaz, A. C., Castillo, A., Sánchez-Matías, M. \& Maass, J. M. (2014). Local values and decisions: Views and constraints for riparian management in western Mexico. Knowledge and Management of Aquatic Ecosystems, 414(6). https://doi.org/10.1051/ $\mathrm{kmae} / 2014017$

Floyd, M. F., Nicholas, L., Lee, I., Lee, J.-H. \& Scott, D. (2006). Social stratification in recreational fishing participation: Research and policy implications. Leisure Sciences, 28(4), 351-368. https://doi.org/10.1080/01490400600745860

Gaspar-Dillanes, M. T. \& Hernández-Montańo, D. (2013). La pesca continental. En M. T. Gaspar-Dillanes \& D. Hernández-Montaño (Eds.), Pesquerías continentales de México (pp. 9-14). México: Instituto Nacional de Pesca (Inapesca). https://doi.org/10.13140/ RG.2.1.1080.8406

Hallwass, G., Lopes, P. F., Juras, A. A. \& Silvano, R. A. M. (2013). Fishers' knowledge identifies environmental changes and fish abundance trends in impounded tropical rivers. Ecological Applications, 23(2), 392-407. https://doi.org/10.1890/12-0429.1

Hallwass, G. \& Silvano, R. A. M. (2016). Patterns of selectiveness in the Amazonian freshwater fisheries: Implications for management. Journal of Environmental Planning and Management, 59(9), 1537-1559. https://doi.org/10.1080/09640568.2015.1081587

Hernández-Sampieri, R., Collado-Ferández, C. \& Baptista-Lucio, P. (2014). Metodología de la investigación (6 $\left.{ }^{\text {ta }} \mathrm{ed}.\right)$. MacGraw-Hill/Interamericana.

Hernández-Morcillo, M., Plieninger, T. \& Bieling, C. (2013). An empirical review of cultural ecosystem service indicators. Ecological Indicators, 29, 434-444. https://doi. org/10.1016/j.ecolind.2013.01.013

Hewlett, B. S. \& Cavalli-Sforza, L. L. (1986). Cultural transmission among Aka Pygmies. American Anthropologist, 88(4), 922-934. http://www.jstor.org/stable/679081

High Level Panel of Experts [HLPE] [Grupo de alto nivel de expertos en seguridad alimentaria y nutrición]/FAO. (2014). La pesca y la acuicultura sostenibles para la seguridad alimentaria y la nutrición. Un informe del Grupo de alto nivel de expertos en seguridad alimentaria y nutrición - Junio 2014. https://www.fao.org/3/a-i3844s.pdf

Infield, M., Anthem, H. \& Morse-Jones, S. (2016). Guidance for the rapid assessment of cultural ecosystem services. Oryx, 50(1), 13-13. https://doi.org/10.1017/ S0030605315001271

Instituto de Información Estadística y Geográfica [IIEG] Jalisco. (2017, julio). Costa-Sierra Occidental. Diagnóstico de la región. www.iieg.gob.mx/contenido/Municipios/09_ costa_sierra_occidental_diagnostico.pdf

Instituto Nacional de Ecología y Cambio Climático [INECC] - Fondo Noroeste [FONNOR], México. (2018). Plan de Acción para el Manejo Integral de Cuencas Hídricas Región Vallarta. Proyecto Conservación de Cuencas Costeras en el Contexto del Cambio Climático.

Instituto Nacional de Estadística y Geografía [INEGI]. (2010). Localidades de la República Mexicana 2010. 
Jiménez de Madariaga, C. \& García del Hoyo, J. J. (2014). Cultura de la pesca, patrimonio y turismo. En J. J. García del Hoyo (Ed.), Cultura, mercados y gestión de la pesca artesanal en el Golfo de Cádiz (pp. 109-131). Universidad de Huelva.

Kals, E., Schumacher, D. \& Montada, L. (1999). Emotional affinity toward nature as a motivational basis to protect nature. Environment and Behavior, 31(2), 178-202. https://doi.org/10.1177/00139169921972056

King, E., Cavender-Bares, J., Balvanera, P., Mwampamba, T. H. \& Polasky, S. (2015). Trade-offs in ecosystem services and varying stakeholder preferences: evaluating conflicts, obstacles, and opportunities. Ecology and Society, 20(3), 25. https://dx.doi. org/10.5751/ES-07822-200325

Kleiber, D., Harris, L. M. \& Vincent, A. C. J. (2014). Gender and small-scale fisheries: A case for counting women and beyond. Fish and Fisheries, 16(4), 1-16. https://doi. org/10.1111/faf.12075

Kruskal, W. H. \& Wallis, W. A. (1952). Use of ranks in One-Criterion Variance Analysis. Journal of the American Statistical Association, 47(260), 583-621. https://doi.org/10.1 080/01621459.1952.10483441

Lau, J. D., Hicks, C. C., Gurney, G. G. \& Cinner, J. E. (2018). Disaggregating ecosystem service values and priorities by wealth, age, and education. Ecosystem Services, 29, 9198. https://doi.org/10.1016/j.ecoser.2017.12.005

Le Roux, B. \& Rouanet, H. (2010). Multiple Correspondence Analysis. sage Publications.

Likert, R. (1932). A technique for the measurement of attitude. Archives of Psychology, 140, 5-55.

Lynch, A. J., Baumgartner, L. J., Boys, C. A., Conallin, J., Cowx, I. G., Finlayson, C. M., Franklin, P. A., Hogan, Z., Koehn, J. D., McCartney, M. P., O’Brien, G. C., Phouthavong, K., Silva, L. G. M., Tob, C. A., Valbo-Jorgensen, J., Vi Vu, A., Whiting, L., Wibowo, A. \& Duncan, P. (2019). Speaking the same language: Can the sustainable development goals translate the needs of inland fisheries into irrigation decisions? Marine and Freshwater Research, 70(9), 1211-1228. https://doi.org/10.1071/MF19176

Lynch, A. J., Cooke, S. J., Deines, A. M., Bower, S. D., Bunnell, D. B., Cowx, I. G., Nguyen, V. M., Nohner, J., Phouthavong, K., Riley, B., Rogers, M. W., Taylor, W. W., Woelmer, W., So-Jung Youn, S. J. \& Douglas, B. T. (2016). The social, economic, and environmental importance of inland fish and fisheries. Environmental Reviews, 24(2), 115-121. https://doi.org/10.1139/er-2015-0064

Lyons, J., González-Hernández, G., Soto-Galera, E. \& Guzmán-Arroyo, M. (1998). Decline of freshwater fishes and fisheries in selected drainages of west-central Mexico. Fisheries, 23(4), 10-18. https://doi.org/10.1577/1548-8446(1998)023<0010:DOF $\mathrm{FAF}>2.0 . \mathrm{CO} ; 2$

McIntyre, P. B., Reidy Liermann, C. A. \& Revenga, C. (2016). Linking freshwater fishery management to global food security and biodiversity conservation. Proceedings of the National Academy of Sciences, 113(45), 12880-12885. https://doi.org/10.1073/ pnas. 1521540113

Mendieta-Izquierdo, G. (2015). Informantes y muestreo en investigación cualitativa. Investigaciones Andina, 17(30), 1148-1150. https://doi.org/10.33132/01248146.65 
Mercado-Silva, N., Santana-Castellón, E., Martínez-Rivera, L., Lyons, J. \& Moermond, T. (2011). Subsistence fisheries in the Sierra Manantlán Biosphere Reserve (Jalisco/Colima, Mexico). e-Gnosis, 9, 1-19. https://www.redalyc.org/articulo.oa?id=73020063007

Millennium Ecosystem Assessment [MA]. (2005). Ecosystems and Human Well-being: Synthesis. Island Press. https://www.millenniumassessment.org/documents/document.356.aspx.pdf

Minitab 17 Statistical Software. (2010). Computer software. www.minitab.com

Nahuelhual, L., Carmona, A., Laterra, P., Barrena, J. \& Aguayo, M. (2014). A mapping approach to assess intangible cultural ecosystem services: The case of agriculture heritage in Southern Chile. Ecological Indicators, 40, 90-101. https://doi.org/10.1016/j. ecolind.2014.01.005

Naiman, R. J. \& Latterell, J. J. (2005). Principles for linking fish habitat to fisheries management and conservation. Journal of Fish Biology, 67(sB), 166-185. https://doi.org/10.1111/ j.0022-1112.2005.00921.x

Naiman, R. J., Decamps, H. \& McClain, M. E. (2010). Riparia: Ecology, conservation, and management of streamside communities. Elsevier Science.

Olaya-Rodríguez, M. H., Escobar Lizarazo, M. D., Cusva, A., Lasso Alcalá, C. A. \& Londoño Murcia, M. C. (2017). Mapeo del servicio ecosistémico de alimento asociado a la pesca en los humedales interiores de Colombia. Ecología Austral, 27, 123-133. https://doi. org/10.25260/EA.17.27.1.1.261

Oleson, K. L. L., Barnes, M., Brander, L. M., Oliver, T. A., van Beek, I., Zafindrasilivonona, B. \& van Beukering, P. (2015). Cultural bequest values for ecosystem service flows among indigenous fishers: A discrete choice experiment validated with mixed methods. Ecological Economics, 114, 104-116. https://doi.org/10.1016/j.ecolecon.2015.02.028

Pascual, U., Balvanera, P., Díaz, S., Pataki, G., Roth, E., Stenseke, M., Watson, R. T., Dessane, E. B., Islar, M., Kelemen, E., Maris, V., Quaas, M., Subramanian, S. M., Wittmer, H., Adlan, A., Ahn, S., Al-Hafedh, Y. S., Amankwah, E., Asah, S. T. ..., Yagi, N. (2017). Valuing nature's contributions to people: the IPBEs approach. Current Opinion in Environmental Sustainability, 26-27, 7-16. https://doi.org/10.1016/j. cosust.2016.12.006

Pauly, D. (1995). Anecdotes and the shifting baseline syndrome of fisheries. Trends in Ecology \& Evolution, 10(10), 430. https://doi.org/10.1016/S0169-5347(00)89171-5

Perea-Bláquez, A. \& Flores Palacios, F. (2016). Participación de las mujeres en la pesca: Nuevos roles de género, ingresos económicos y doble jornada. Sociedad y Ambiente, 1(9), 121141. https://doi.org/10.31840/sya.v0i9.1636

Pollnac, R. B. \& Poggie, J. J. (2008). Happiness, well-being, and psychocultural adaptation to the stresses associated with marine fishing. Human Ecology Review, 15(2), 194-200. http://www.apjh.humanecologyreview.org/pastissues/her152/pollnacpoggie.pdf

Pomeroy, R. (2016). A research framework for traditional fisheries: Revisited. Marine Policy, 70, 153-163. https://doi.org/10.1016/j.marpol.2016.05.012

Pope, K. L., Pegg, M. A., Cole, N. W., Siddons, S. F., Fedele, A. D., Harmon, B. S., Ruskamp, R., Turner, D. \& Uerling, C. C. (2016). Fishing for ecosystem services. Journal of Environmental Management, 183(2), 408-417. https://doi.org/10.1016/j. jenvman.2016.04.024

Pyle, R. M. (1993). The thunder tree: lessons from an urban wildland. Houghton Mifflin. 
Raymond, C. M., Bryan, B. A., MacDonald, D. H., Cast, A., Strathearn, S., Grandgirard, A. \& Kalivas, T. (2009). Mapping community values for natural capital and ecosystem services. Ecological Economics, 68(5), 1301-1315. http://dx.doi.org/10.1016/j. ecolecon.2008.12.006

Rodrigues, J. M. G. (2015). Cultural services in aquatic ecosystems. En L. Chicharo, F. Müller \& N. Fohrer (Eds.), Ecosystem Services and River Basin Ecohydrology (pp. 35-56). Springer.

Rojas-Soriano, R. (2013). Guía para realizar investigaciones sociales (38 ed.). Plaza y Valdés, S.A. de C.V.

Sáenz-Arroyo, A., Roberts, C. M., Torre, J., Cariño-Olvera, M. \& Enríquez-Andrade, R. R. (2005). Rapidly shifting environmental baselines among fishers of the Gulf of California. Proceedings of the Royal Society London, Series B Biological Sciences, 272, 1957-1962. https://doi.org/10.1098/rspb.2005.3175

Santha, S. D. (2008). Local ecological knowledge and fisheries management: A study among riverine fishing communities in Kerala, India. Local Environment, 13(5), 423-435. https://doi.org/10.1080/13549830701809726

Schafer, A. G. \& Reis, E. G. (2008). Artisanal fishing areas and traditional ecological knowledge: The case study of the artisanal fisheries of the Patos Lagoon estuary (Brazil). Marine Policy, 32(3), 283-292. https://doi.org/10.1016/j.marpol.2007.06.001

Schnegg, M., Rieprich, R. \& Pröpper, M. (2014). Culture, nature, and the valuation of ecosystem services in Northern Namibia. Ecology and Society, 19(4), 26. http://dx.doi. org/10.5751/ES-06896-190426

Sharma, C. (2011). Securing economic, social and cultural rights of small-scale and artisanal fisherworkers and fishing communities. MAST, 10(2), 41-61. http://www.marecentre. $\mathrm{nl} /$ mast/documents/MAST10.2_Sharma.pdf

Small, N., Munday, M. \& Durance, I. (2017). The challenge of valuing ecosystem services that have no material benefits. Global Environmental Change, 44, 57-67. https://doi. org/10.1016/j.gloenvcha.2017.03.005

Soga, M. \& Gaston, K. J. (2016). Extinction of experience: The loss of human-nature interactions. Frontiers in Ecology and the Environment, 14(2), 94-101. https://doi. org/10.1002/fee.1225

Syrbe, R.-U., Schröter, M., Grunewald, K., Walz, U. \& Burkhard, B. (2017). Mapping ecosystem services. En B. Burkhard \& J. Maes (Eds.), Advanced Books (Vol. 1, pp. 151-158). Pensoft Publishers.

Villamagna, A. M., Mogollon, B. \& Angermeier, P. L. (2014). A multi-indicator framework for mapping cultural ecosystem services: The case of freshwater recreational fishing. Ecological Indicators, 45, 255-265. https://doi.org/10.1016/j.ecolind.2014.04.001

Weeratunge, N., Béné, C., Siriwardane, R., Charles, A., Johnson, D., Allison, E. H., Nayak P. K. \& Badjeck, M.-C. (2014). Small-scale fisheries through the wellbeing lens. Fish and Fisheries, 15(2), 255-279. https://doi.org/10.1111/faf.12016

World Bank, FAO [Food and Agricultural Organization] \& IFAD [International Fund for Agricultural Development]. (2008). Module 13 Gender in Fisheries and Aquaculture. En Gender in Agriculture Sourcebook (pp. 561-599). World Bank Publications. 